\title{
Refractory status epilepticus after inadvertent intrathecal injection of tranexamic acid treated by magnesium sulfate
}

\author{
D.M. Hatch, E. Atito-Narh ${ }^{\S}$ E.J. Herschmiller, ${ }^{\dagger}$ A.J. Olufolabi, ${ }^{\dagger}$ M.D. Owen \\ Department of Anesthesiology, Wake Forest School of Medicine, Winston-Salem, NC, USA \\ Department of Anesthesiology, Ridge Regional Hospital, Accra, Ghana \\ Department of Anesthesiology, Duke University Medical Center, Durham, NC, USA
}

\begin{abstract}
We present a case of accidental injection of tranexamic acid during spinal anesthesia for an elective cesarean delivery. Immediately following intrathecal injection of $2 \mathrm{~mL}$ of solution, the patient complained of severe back pain, followed by muscle spasm and tetany. As there was no evidence of spinal block, the medications given were checked and a 'used' ampoule of tranexamic acid was found on the spinal tray. General anesthesia was induced but muscle spasm and tetany persisted despite administration of a non-depolarizing muscle relaxant. Hemodynamic instability, ventricular tachycardia, and status epilepticus developed, which were refractory to phenytoin, diazepam, and infusions of thiopental, midazolam and amiodarone. Magnesium sulfate was administered postoperatively in the intensive care unit, following which the frequency of seizures decreased, eventually stopping. Unfortunately, on postoperative day three the patient died from cardiopulmonary arrest after an oxygen supply failure that was not associated with the initial event. This report underlines the importance of double-checking medications before injection in order to avoid a drug error. As well, it suggests that magnesium sulfate may be useful in stopping seizures caused by the intrathecal injection of tranexamic acid.
\end{abstract}

(C) 2015 Elsevier Ltd. All rights reserved.

Keywords: Cesarean section; Spinal anesthesia; Tranexamic acid; Magnesium; Medication error

\section{Introduction}

Several factors have been identified as contributing to medication errors in anesthesia, including similar appearance or location of vials, miscommunication, anesthesia provider inattention, and fatigue. ${ }^{1-3}$ While the true frequency of anesthesia-related medication errors is unknown, these errors are an increasing global health problem. ${ }^{1-3}$ We describe the accidental intrathecal tranexamic injection in a low-resource setting that resulted in seizures that were refractory to standard therapy. Subsequent management and use of

\footnotetext{
Accepted November 2015

Correspondence to: D. Matt Hatch, MD, Department of Anesthesiology, Wake Forest School of Medicine, Medical Center Boulevard, Winston-Salem, NC 27157-1009, USA.

E-mail address: dhatch@wakehealth.edu

${ }^{\S}$ Current address for Dr E. Atito-Narh: Department of Anesthesiology, Ridge Regional Hospital, PO Box 473, Accra, Ghana.

${ }^{\dagger}$ Current address for Dr E. J. Herschmiller: Department of Anesthesiology, New York-Presbyterian, 630 West 168th Street, New York, NY 10032, USA.

${ }^{\ddagger}$ Current address for Dr A. J. Olufolabi: Department of Anesthesiology, Duke University Medical Center, Box 3094 Med Ctr, Durham, NC 27710, USA.
}

magnesium sulfate is discussed along with the initiation of a new operating room protocol for drug administration in an attempt to minimize the risk of this complication.

\section{Case report}

A 31-year-old, G3P2 woman was admitted to a West African regional hospital at 33 weeks of gestation with premature rupture of membranes and pyelonephritis. She had no significant past medical, obstetric or anesthesia history other than two previous cesarean deliveries. She was treated with erythromycin and metronidazole. Two doses of steroids were administered for fetal lung maturity. Two days after admission, she developed abdominal tenderness and intravenous cefuroxime was started. The patient was scheduled for repeat cesarean delivery eight days after admission due to worsening pyelonephritis.

She received intravenous lactated Ringer's solution $1 \mathrm{~L}$ before arriving in the operating room. An anesthesia technician was assisting the nurse anesthetist providing care for the case. The technician opened a glass vial of 
medication and held it away from the sterile field while the nurse anesthetist drew up $2 \mathrm{~mL}$ of solution. Lumbar puncture was performed with the patient in a sitting position, at the L4-5 interspace with a 25 -gauge Whitacre needle. Shortly after injection, the patient complained of severe burning back pain, followed by lower extremity spasms and difficulty in lying supine due to tetany. After failure to achieve anesthesia, the team prepared to repeat the intrathecal injection at which point an opened vial of tranexamic acid was discovered $(100 \mathrm{mg} / \mathrm{mL})$ (Fig. 1). The consultant anesthesiologist was notified and a second spinal was not attempted. General anesthesia was induced with propofol $150 \mathrm{mg}$ and suxamethonium $100 \mathrm{mg}$; the patient was intubated and anesthesia maintained with an uncharted dose of volatile anesthetic. Due to ongoing myoclonus and tetany, vecuronium $4 \mathrm{mg}$ was administered shortly after induction. The patient remained tachycardic and hypertensive with heart rates of $120-150$ beats/min and a blood pressure of $165 / 105 \mathrm{mmHg}$ throughout the case with occasional short intervals of ventricular tachycardia. Treatment with amiodarone $150 \mathrm{mg}$, midazolam (dose unknown), diazepam $10 \mathrm{mg}$, and labetalol $20 \mathrm{mg}$ were unsuccessful in treating the tachycardia and hypertension or terminating seizures.

A live, male infant was delivered weighing $1.6 \mathrm{~kg}$ with Apgar scores of 7 and 8 at 1- and $5 \mathrm{~min}$, respectively. The case was completed within one hour of the initial intrathecal injection and the patient, who remained intubated and ventilated, was taken to the intensive care unit whilst receiving midazolam $(2 \mathrm{mg} / \mathrm{h}), 10 \%$ mannitol $100 \mathrm{~mL} / \mathrm{h}$, amiodarone (unknown rate), and fentanyl $200 \mu \mathrm{g} / \mathrm{h}$. Upon arrival, her heart rate was documented

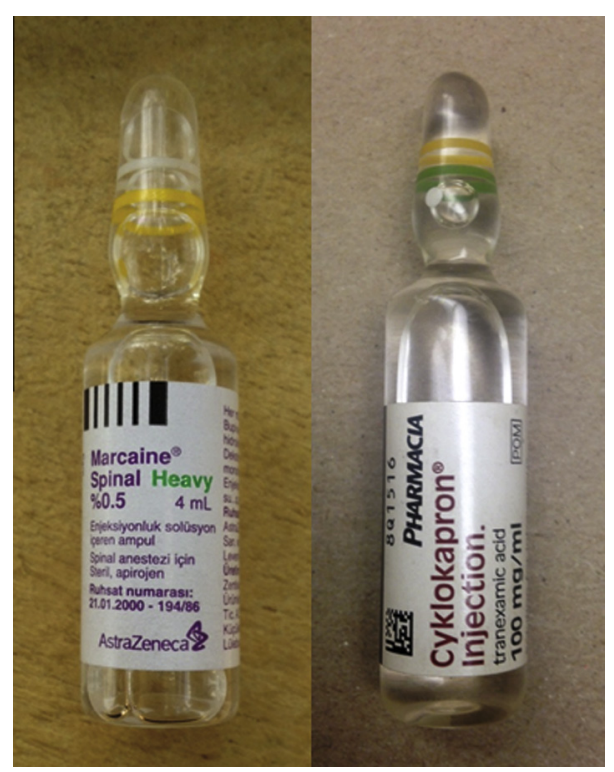

Fig. 1 Ampules of bupivacaine and tranexamic acid. The similar size of ampules led to a medication administration error during spinal anesthesia at 164 beats/min and blood pressure 225/164 $\mathrm{mmHg}$. She continued "twitching" per handwritten charts and was treated with vecuronium $4 \mathrm{mg}$, thiopental $150 \mathrm{mg}$ followed by phenytoin $100 \mathrm{mg}$ eight hourly, dexamethasone $8 \mathrm{mg}$ eight hourly, vecuronium $10 \mathrm{mg} / \mathrm{h}$, thiopental $500 \mathrm{mg} / \mathrm{h}$ and pethidine $50 \mathrm{mg}$ four hourly. Laboratory values obtained showed a hemoglobin value of $12.6 \mathrm{~g} / \mathrm{dL}$, white blood cell count of $21.1 \times 10^{9} / \mathrm{L}$, and a platelet count of $356 \times 10^{9} / \mathrm{L}$.

Her vital signs remained unstable throughout the evening requiring placement of a radial arterial line and a central venous line. There were repeated bursts of ventricular tachycardia that spontaneously converted to sinus rhythm without medical intervention. Seizure activity continued despite intravenous boluses of diazepam, dexamethasone, and phenytoin and infusions of midazolam, fentanyl, mannitol, vecuronium, and thiopental. As she had ongoing seizure activity, despite antiepileptic or benzodiazepine boluses, the vecuronium and thiopental infusions were discontinued.

On postoperative day two, the anesthesiologist ordered magnesium sulfate ( $4 \mathrm{~g}$ bolus) followed by an infusion of $1 \mathrm{~g} / \mathrm{h}$. This had an immediate effect of reducing the seizure frequency. Within hours, the seizures terminated altogether and the patient was able to open her eyes and move all of her extremities as well as make independent respiratory effort while on the ventilator. Midazolam and fentanyl infusions were gradually decreased and boluses of these two drugs were only used as needed. A serum magnesium level was ordered but the result was never obtained.

While showing many signs of clinical improvement without seizure activity such as purposeful movement in her extremities, eye opening, and respiratory effort, the patient remained in the intensive care unit on mechanical ventilation due to need for continued respiratory support. Unfortunately, on day three the main oxygen supply of the hospital stopped working and the patient desaturated and went into cardiopulmonary arrest. As the oxygen issue was being resolved, and she was pronounced dead.

\section{Discussion}

In our case, an accidental injection of tranexamic acid, instead of bupivacaine, for spinal anesthesia led to refractory seizure activity. Factors thought to be responsible for the mistake were human error and the fact that the two vials were of similar size and color.

This case is an example of a mistake occurring in a low-resource setting due to human error and the lack of a system to help prevent medication errors. In lowresource countries the actual number of medication errors is vastly understated and remains a key problem. ${ }^{2,3}$ Failure to report medication errors in lowresource environments can occur for a variety of reasons 
including poor documentation, inattentiveness, inadequate staff or equipment, reliance on paper charting which can be lost, and poor handwriting. ${ }^{1-3}$ Difficulties encountered in reporting this case included poor documentation and lack of laboratory assessment. The North American authors were not physically present during this case; however, they are part of the supportive and consultative role that is maintained with staff in this hospital through Kybele, Inc. ${ }^{4}$ The North American authors were notified of this occurrence and a joint review of the case was conducted.

Tranexamic acid is an antifibrinolytic that competitively inhibits plasminogen-binding sites and decreases plasmin formation and fibrinolysis. It is used primarily to decrease bleeding complications following orthopedic, gynecologic and cardiac surgery. However, if administered incorrectly, such as intrathecally, tranexamic acid can produce serious complications. There are several reports similar to ours with some cases proving fatal. ${ }^{5-11}$ Wong et al. first described accidental intrathecal administration of tranexamic acid when a $75 \mathrm{mg}$ injection resulted in seizures that resolved with diazepam and without neurologic sequelae. ${ }^{5}$ Other reports have followed with intrathecal doses ranging from $75-500 \mathrm{mg}$ with similar presentations including severe back pain followed by tetany, hypertension, tachycardia, and cardiac arrhythmias with both fatal and non-fatal outcomes. ${ }^{6-11}$

Treatment in each case followed two similar pathways: management of seizures and of cardiac arrhythmias. Most cases applied a combination of antiepileptic medication including benzodiazepines, barbiturates, phenytoin, sodium valproate, mannitol, levetiracetam, dexamethasone and even non-depolarizing muscle relaxants with varying success. No reports to date have utilized magnesium sulfate. Treatment of cardiac arrhythmias included lidocaine, amiodarone, nitroglycerin, beta blockade, and cardioversion/defibrillation. While not unexpected, there does appear to be a correlation of prolonged seizures, increased cardiac arrhythmias, and mortality with increasing doses of intrathecal tranexamic acid. Tranexamic acid should never be administered by intrathecal injection.

Looking specifically at the drug and its unintended side effects, Murkin et al. reported that high dose intravenous tranexamic acid (61-259 mg/kg) used during cardiac surgery increased postoperative seizures from $1.3 \%$ to $3.8 \%{ }^{12}$ While no human trials have been conducted, Yamamura et al. reported that $5 \mathrm{mg} / \mathrm{kg}$ of intrathecal tranexamic acid produced seizures within 45-60 seconds in felines with a ruptured intracranial aneurysm. ${ }^{13}$

The proposed mechanisms for the cardiac and neurologic manifestations include central sympathetic discharge, $\mathrm{GABA}_{\mathrm{A}}$ inhibition, and direct cerebral ischemia. ${ }^{6-11,14}$ In a report similar to ours, a patient was inadvertently given $500 \mathrm{mg}$ intrathecal tranexamic acid and developed convulsions followed by a pulseless electrical activity arrest treated with diazepam, multiple defibrillation attempts, epinephrine, and amiodarone. ${ }^{6}$ Her seizures were successfully terminated with diazepam; however, she developed refractory ventricular fibrillation, cardiac collapse and died. The authors suggested that tranexamic acid produced massive sympathetic discharge, which resulted in hypertension, tachycardia, and tonic-clonic seizures. ${ }^{6}$ Our case involved a high intrathecal dose and resulted in mortality, although our patient's demise appeared to have been related to a separate medical error.

Another hypothesis is that seizures result from blockade of the inhibitory cortical $\mathrm{GABA}_{\mathrm{A}}$ receptors. ${ }^{7}$ Butala et al. described an accidental $300 \mathrm{mg}$ tranexamic acid injection for spinal anesthesia for cystolithotripsy. ${ }^{7}$ The patient also developed back pain and myoclonic jerks. Multiple anticonvulsants were administered and were successful in terminating seizure activity after several days. She was later discharged without neurologic sequelae. The authors reasoned that tranexamic acid blockade of $\mathrm{GABA}_{\mathrm{A}}$ receptors lower the depolarization threshold and enhanced excitotoxicity through chloride channels. This has been echoed by other authors. ${ }^{8,14}$

A third hypothesis is that tranexamic acid causes seizures through direct cerebral hypoperfusion. Mahmoud and Ammar described tranexamic acid $300 \mathrm{mg}$ given accidentally as a spinal dose for lower extremity skin grafting. ${ }^{10}$ The patient developed myoclonic seizures and ventricular fibrillation that responded to midazolam, propofol, defibrillation, propranolol, and amiodarone, respectively. $\mathrm{He}$ was discharged without neurologic sequelae. The authors suggested that cerebral ischemia due to vasoconstriction could account for the presentation.

Whatever the hypothesis, the result appears to be the same: myoclonic seizures with cardiac arrhythmias. Several cases resulted in neurologic recovery with patients safely discharged. ${ }^{5,7,8,10,11,13}$ In other cases, ours included, the patient died. ${ }^{6,9}$ Our case presented an interesting dilemma in that, despite treatment with benzodiazepines, barbiturates and phenytoin, our patient remained in refractory status epilepticus until magnesium was added.

Magnesium sulfate is a useful medication for treating multiple conditions such as pain, torsades de pointes, hypertension, eclampsia, and neuroprotection. ${ }^{15}$ Among its many actions, it has a central nervous system depressant effect as an N-nitrosodimethylamine (NMDA) receptor antagonist and an inhibitor of catecholamine release. Both of these pathways could explain how magnesium benefited our patient. There is evidence that seizures are mediated through NMDA receptors, so an antagonist could inhibit seizure activity. ${ }^{15,16}$ Magnesium is administered for the prevention and treatment of eclamptic seizures. ${ }^{16}$ Mechanisms thought to be 


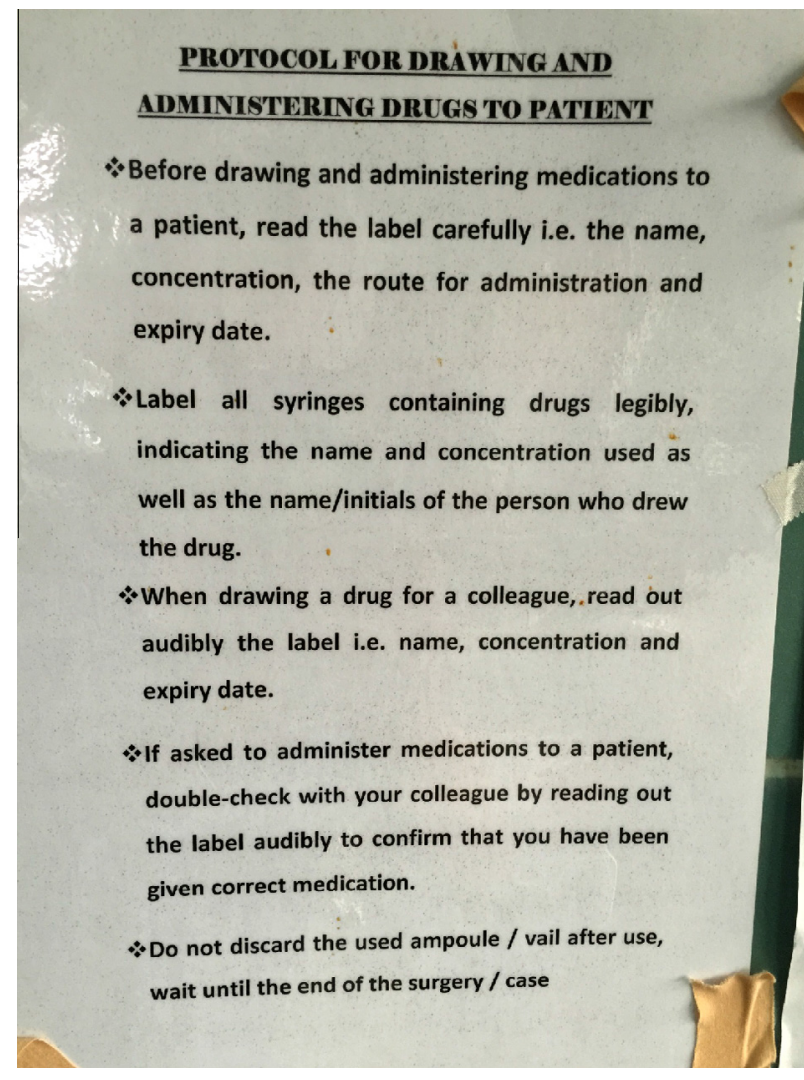

Fig. 2 The new protocol for drug administration in operating rooms at the Ridge Regional Hospital, Accra, Ghana implemented after this case

involved in the treatment of eclampsia include vasodilation, blood brain barrier protection to decrease cerebral edema and central anticonvulsant effects. ${ }^{16}$ The direct inhibition of catecholamine release could also explain magnesium's anticonvulsant effect on our patient if the hypothesis of direct sympathetic discharge is accepted. Magnesium produces vasodilatation through calcium antagonism in vascular smooth muscle. ${ }^{15}$ Thus, if cerebral vasoconstriction is involved in producing global ischemia, magnesium could promote blood flow.

Magnesium has also been applied for the treatment of cardiac arrhythmias. Although not used routinely in Advanced Cardiac Life Support algorithms, it has been used for malignant cardiac arrhythmias, such as torsades de pointes. ${ }^{15}$ Studies have shown that magnesium slows electrical activity in the SA node, prolongs AV conduction, and increases the refractory period of the AV node. ${ }^{15}$ Several patients experienced cardiac arrhythmias after accidental spinal tranexamic acid administration which were refractory to defibrillation. ${ }^{6,9}$ Magnesium sulfate might have been useful in stabilizing the cardiac arrhythmias in these patients.

It is unfortunate that errors occur in medicine. Incorrect drug administration can produce devastating results so anesthesia providers must be vigilant when administering medication, even when packaging is dissimilar. In our case, two similar sized vials were mistaken despite dissimilar writing with the results that the patient received an unintended medication with unfavorable consequences. Oxygen supply failure and inadequate critical care capacity, which also contributed to the patient's demise, are relatively common in lowresource settings and fall outside the scope of this paper. After our joint review, the hospital implemented a protocol whereby every medication is double-checked and read back by two providers to confirm verbally which medication is being administered (Fig. 2). The hospital also changed brands of bupivacaine with new labeling. These changes will hopefully reduce the risk of similar complications occurring in the future.

In addition to the description of the medication error, this report describes a potentially novel use of magnesium sulfate. Although administered as a treatment for other types of seizures, this is the first report of magnesium being used to treat seizures related to the intrathecal injection of tranexamic acid.

\section{Disclosure}

Funding for Dr Hatch was provided, in part, by the Society for Obstetric Anesthesia and PerinatologyKybele International Outreach Grant.

\section{References}

1. Wheeler SJ, Wheeler DW. Medication errors in anaesthesia and critical care. Anaesthesia 2005;60:257-73.

2. Kouffer GA, Anto BP, Abaitey AK. Error-provoking conditions in the medication use process: the case of a government hospital in Ghana. J Patient Saf 2012;8:22-5.

3. Nwasor EO, Sule ST, Mshelia DB. Audit of medication errors by anesthetists in North Western Nigeria. Niger $J$ Clin Pract 2014; 17:226-31.

4. Olufolabi AJ, Atito-Narh E, Eshun M, Ross VH, Muir HA, Owen MD. Teaching neuraxial techniques for obstetric care in a Ghanaian referral hospital: achievements and obstacles. Anesth Analg 2015;120:1317-22.

5. Wong JO, Yang SF, Tsai MH. Accidental injection of tranexamic acid (Transamin) during spinal anesthesia. Ma Zui Xue Za Zhi 1988;26:249-52.

6. Yeh HM, Lau HP, Lin PL, Sun WZ, Mok MS. Convulsions and refractory ventricular fibrillation after intrathecal injection of a massive dose of tranexamic acid. Anesthesiology 2003;98:270-2.

7. Butala BP, Shah VR, Bhosale GP, Shah RB. Medication error: subarachnoid injection of tranexamic acid. Indian $J$ Anaesth 2012:56:168-70.

8. Kaabachi O, Eddhif M, Rais K, Zaabar MA. Inadvertent intrathecal injection of tranexamic acid. Saudi $J$ Anaesth 2011;5:90-2.

9. Garcha PS, Mohan CV, Sharma RM. Death after an inadvertent intrathecal injection of tranexamic acid. Anesth Analg 2007; 104:241-2.

10. Mahmoud K, Ammar A. Accidental intrathecal injection of tranexamic acid. Case Rep Anesthesiol 2012. http://dx.doi.org/ $10.1155 / 2012 / 646028$ 
11. Mohseni K, Jafari A, Nobahar MR, Arami A. Polymyoclonus seizure resulting from accidental injection of tranexamic acid in spinal anesthesia. Anesth Analg 2009;108:1984-6.

12. Murkin JM, Falter F, Granton J, Young B, Burt C, Chu M. Highdose tranexamic acid is associated with nonischemic clinical seizures in cardiac surgical patients. Anesth Analg 2010;110:350-3.

13. Yamaura A, Nakamura T, Makino H, Hagihara Y. Cerebral complication of antifibrinolytic therapy in the treatment of ruptured intracranial aneurysm. Animal experiment and a review of literature. Eur Neurol 1980;19:77-84.
14. Furtmüller R, Schlag MG, Berger M, et al. Tranexamic acid, a widely used antifibrinolytic agent, causes convulsions by a gammaaminobutyric acid(A) receptor antagonistic effect. J Pharmacol Exp Ther 2002;301:168-73.

15. Herroeder S, Schönherr ME, De Hert SG, Hollmann MW. Magnesium-essentials for anesthesiologists. Anesthesiology 2011;114:971-93.

16. Euser AG, Cipolla MJ. Magnesium sulfate for the treatment of eclampsia: a brief review. Stroke 2009;40:1169-75. 